\title{
Selectivity and Activity of Iron Molybdate Catalysts in Oxidation of Methanol
}

\author{
Khalid Khazzal Hummadi*1, Karim H. Hassan² and Phillip C.H. Mitchell ${ }^{3}$ \\ ${ }^{*}$ Al Khawarizmi College of Engineering, University of Baghdad, Al. Jadiria, Baghdad, Iraq \\ ${ }^{2}$ Ministry of Science and Technology, Al. Jadiria, Baghdad, Iraq \\ ${ }^{3}$ School of Chemistry, University of Reading, Reading RG6 6AD, UK
}

Received 29 March 2006; accepted 21 April 2007
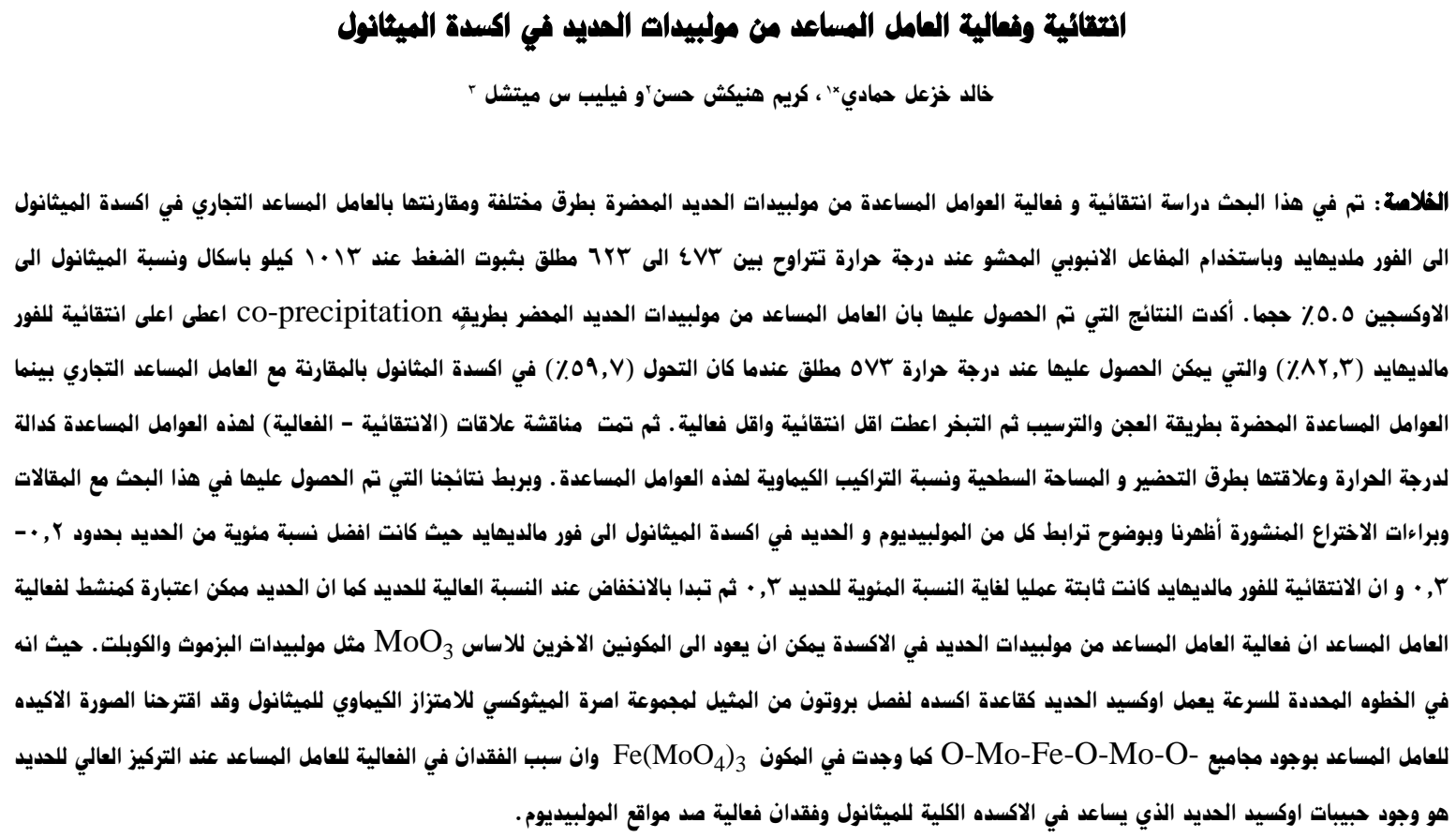

Abstract: The selectivity and activity of iron molybdate catalysts prepared by different methods are compared with those of a commercial catalyst in the oxidation of methanol to formaldehyde in a continuous tubular bed reactor at $200-350{ }^{\circ} \mathrm{C}$ (473-623 $\left.{ }^{\circ} \mathrm{K}\right), 10 \mathrm{~atm}(1013 \mathrm{kPa})$, with a methanol-oxygen mixture fixed at 5.5\% by volume methanol: air ratio. The iron(III) molybdate catalyst prepared by co-precipitation and filtration had a selectivity towards formaldehyde in methanol oxidation comparable with a commercial catalyst; maximum selectivity $(82.3 \%)$ was obtained at $573^{\circ} \mathrm{K}$ when the conversion was $59.7 \%$. Catalysts prepared by reacting iron (III) and molybdate by kneading or precipitation followed by evaporation, omitting a filtration stage, were less active and less selective. The selectivity-activity relationships of these catalysts as a function of temperature were discussed in relation to the method of preparation, surface areas and composition. By combing this catalytic data with data from the patent literature we demonstrate a synergy between iron and molybdenum in regard to methanol oxidation to formaldehyde; the optimum composition corresponded to an iron mole fraction 0.2-0.3. The selectivity to formaldehyde was practically constant up to an iron mole fraction 0.3 and then decreased at higher iron concentrations. The iron component can be regarded as the activity promoter. The iron molybdate catalysts can thus be related to other two-component $\mathrm{MoO}_{3}$-based selective oxidation catalysts, e.g. bismuth and cobalt molybdates. The iron oxide functions as a relatively basic oxide abstracting, in the rate-controlling step, a proton from the methyl of a bound methoxy group of chemisorbed methanol. It was proposed that a crucial feature of the sought after iron(III) molybdate catalyst is the presence of -O-Mo-O-Fe-O-Mo-O- groups as found in the compound $\mathrm{Fe}_{2}\left(\mathrm{MoO}_{4}\right)_{3}$ and for $\mathrm{Fe}^{3+}$ well dispersed in $\mathrm{MoO}_{3}$ generally. At the higher iron(III) concentrations the loss of selectivity is due to the presence of iron oxide patches or particles which catalyze the total oxidation of methanol, and the loss of activity to blocking of molybdenum sites.

Keywords: Selective oxidation, Methanol, Formaldehyde, Iron molybdate, Activity 


\section{Introduction}

Many researches deal with the oxidation of methanol using many different catalysts to produce the formaldehyde which has many important industrial chemical applications in the production of urea, melamine, phenolic resins and ethylene glycol; in the fertilizer and paints industries; and in medicine as a preservative (See, for example, The Formaldehyde Council, Inc. (FCI): Formaldehyde.org). Formaldehyde is manufactured by the selective oxidation of methanol with oxygen or air over a silver (Jia-Linang Li, et al. 2000; Wachs, I.E., 2003) or iron molybdate catalysts (Farrauto, et al. 1997; Soares, et al. 2001; and Ivanov, et al. 2000). Iron molybdate is a typical two-component selective oxidation catalyst. The two components, iron(III) oxide and molybdenum(VI) oxide, interact to produce a catalyst which favours the desired product, formaldehyde, rather than the products of total oxidation of methanol, carbon dioxide and water, or other products like carbon monoxide or dimethyl ether. It is the combination of the two oxides which produces the desired active and selective catalyst. Iron (III) oxide by itself is unselective producing carbon dioxide and water; molybdenum trioxide is selective but with low activity (Bowker, et al. 2002 and Wang, et al. 2001).

The overall reaction is

$$
\mathrm{CH}_{3} \mathrm{OH}+0.5 \mathrm{O}_{2}=\mathrm{CH}_{2} \mathrm{O}+\mathrm{H}_{2} \mathrm{O}
$$

The reaction is exothermic $\left(\Delta H,=-159 \mathrm{~kJ} \mathrm{~mol}^{-1}\right)$ (Diakov, et al. 2002 and Dia Kov, et al. 2001). The oxidation proceeds through reaction of methanol with the molybdate surface (McCarron III, et al. 1986). At ambient temperature methoxy groups are formed and water is liberated. As the temperature is raised the methoxy groups begin to decompose forming formaldehyde and more water. The rate-limiting step is the abstraction of methoxy hydrogen via breaking of a $\mathrm{C}-\mathrm{C}$ bond. The products rapidly desorbed leaving the molybdate phase reduced. Re-oxidation is affected by oxygen so completing the catalytic cycle. The active sites are considered to be co-ordinatively unsaturated molybdenum atoms. The overall reaction is in accordance with the Mars-van Krevelen mechanism, i.e. oxygen is supplied to the reactant as lattice oxygen rather than by direct reaction with oxygen. The lattice oxygen is replenished by reactant oxygen.

The technical catalyst composition is $80 \% \mathrm{MoO}_{3}$ and $20 \% \mathrm{Fe}_{2} \mathrm{O}_{3}$ equivalent to an iron mole fraction 0.31 . Iron may be partially replaced by a promoter, eg. chromium. The active catalyst is considered to be $\mathrm{Fe}_{2}\left(\mathrm{MoO}_{4}\right)_{3}$ (Soares, et al. 2001). The excess of $\mathrm{MoO}_{3}$ is said variously to be required to ensure the stability of the catalyst towards loss of $\mathrm{MoO}_{3}$, to maintain the active species and to enhance the surface area (Soares, et al. 2001). We shall see that it may be more profitable to describe the catalyst as $\mathrm{Fe}^{3+}$ dispersed in $\mathrm{MoO}_{3}$.

The chemical composition is the major factor determin- ing the catalytic properties. However the catalytic characteristics depend on catalyst preparation method, reflecting changes in the extent of interaction of the iron and molybdenum oxides, the extent of dispersion, the surface area, the pore structure, and the crystal structure (Boreskov, 1976). It should be stressed that catalyst structure depends also on other parameter such as metal loading, surface area of the carrier and drying and calcination temperatures (Topsoe, et al. 1976). The method of preparation appears to have a significant impact on the activity and selectivity of the catalyst. There have been some studies of different preparation methods, for example sol-gel catalysts by co-precipitated catalysts (Wand, et al. 2001; Soares, et al. 1997; and Soares, 2003). In the present work iron molybdate catalysts were prepared by three methods-kneading or slurry method, precipitation and evaporation, co-precipitation and filtration. The activities and selectivities in the oxidation of methanol were determined at $473-623^{\circ} \mathrm{K}$ and compared with the activity and selectivity of a commercial catalyst.

\section{Experimental Work}

\subsection{Catalyst Preparation}

Ammonium molybdate, $\left(\mathrm{NH}_{4}\right)_{6} \mathrm{Mo}_{7} \mathrm{O}_{24} \cdot 4 \mathrm{H}_{2} \mathrm{O}$; and iron (III) nitrate nonahydrate, $\mathrm{Fe}\left(\mathrm{NO}_{3}\right)_{3} 9 \mathrm{H}_{2} \mathrm{O}$, analytical grade (>99\% purity), were used.

\subsubsection{Kneading and Evaporation: Catalyst (1)}

To prepare $25 \mathrm{~g}$ of catalyst, $24.2 \mathrm{~g}$ of ammonium molybdate was added to amount of distilled water which is enough so that when added to $25.31 \mathrm{~g}$ of iron nitrate powder gradually with continuous stirring to obtain a homogenous paste. The paste was dried in an oven at $383^{\circ} \mathrm{K}$ for 2 hours to evaporate water and then calcined at 673$773^{\circ} \mathrm{K}$ in a current of air for 4 hour. The calcined catalyst was crushed to small pieces, then to powder using a ball mill. The 100 mesh fraction was mixed with a solution of polyvinyl alcohol (3\%) in distilled water. The produced paste was then dried, crushed, and sieved. Pellets (or tablets) (7 mm diameter, $4 \mathrm{~mm}$ thick) were prepared in a tablet press at $2 \mathrm{~atm}$. The pellets were calcined at $773^{\circ} \mathrm{K}$ to dissociate the polyvinyl alcohol because it is used as a binder only.

\subsubsection{Precipitation and Concentration: Catalyst (2)}

Solutions of ammonium molybdate, $24.52 \mathrm{~g}$ in $250 \mathrm{ml}$ of distilled water and iron(III) nitrate $25.31 \mathrm{~g}$ in $150 \mathrm{ml}$ of distilled water were mixed, the $\mathrm{pH}$ was $\mathrm{ca} 2$. The precipitate formed was left to settle overnight at room temperature to get rid of excess water and then dried, calcined and formulated as for catalyst 1 .

\subsubsection{Co-precipitation and Filtration: Catalyst (3)}

Solutions of ammonium molybdate and iron(III) nitrate were prepared and mixed as in the preparation of catalyst 2. The precipitate was filtered off and washed several times with distilled water until the $\mathrm{pH}$ of the filtrate reached 7 . The solid was dried, calcined and formulated 

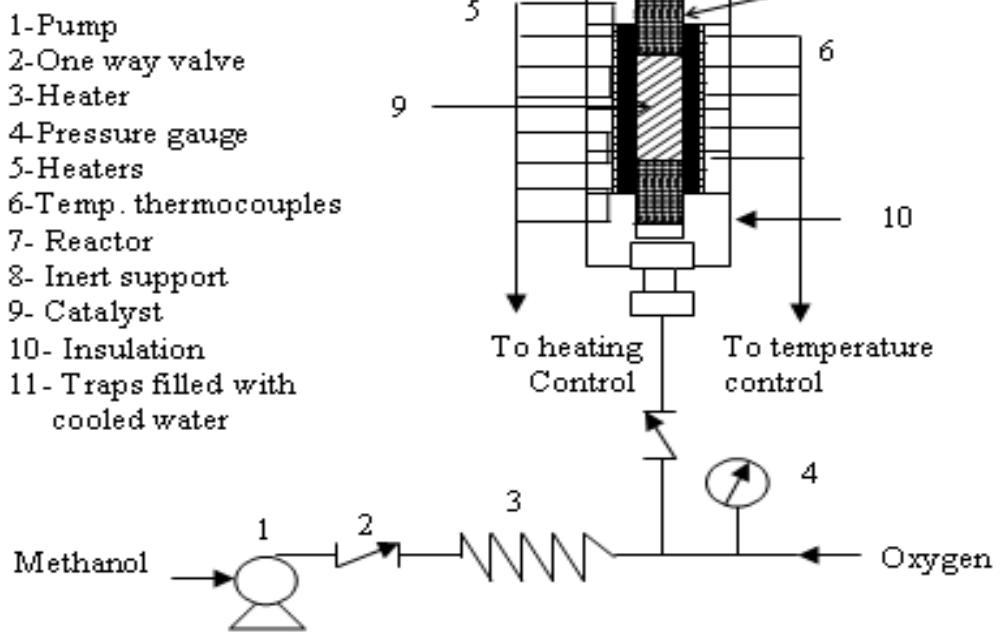

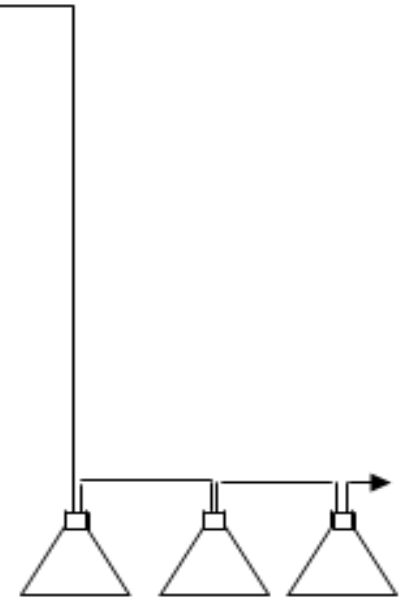

11

Figure 1. Flow diagram of laboratory oxidation of methanol unit

as before.

\subsection{Catalyst Characterization}

The analysis of Iron and molybdenum were determined by standard atomic absorption spectrophotometer (PYE UNICAM SP9).

The X-ray diffraction of powder of prepared and commercial catalysts were carried out by using a Phillips Xray diffractometer with $\mathrm{CuK}_{\alpha}$ radiation $(1.542 \AA, 40 \mathrm{kV}$, $20 \mathrm{~mA}$ ) scanning over the range of angles of 3 to 70 on 2. Peaks were assigned by comparison with the $d$ spacing of typical compounds given in the ASTM powder diffraction files (Powder Diffraction File, Swarthmore Pennsylvania, 1978).

Surface areas of the catalysts were determined by the BET method (Le Page, 1987) (carloEraba sorptomic series 1800). Pore volumes and densities were determined by the liquid impregnation method (Satter Filled and Charles, 1980). Hardnesses were determined with the ERWEKA TBH28 hardness meter (Le Page, 1987).

The bulk density is determined by replacing a weight catalyst in a graduated cylinder then shake it by hand on the table or by vibrator and read the volume of it and then calculate the density, it is the packing or load density of the reactor. The solid density is measured by weighting a symmetrical tablet and calculates its volume. It is carried out for several tablets and takes the average.

\subsection{Catalyst Testing}

Activities and selectivities of the catalysts in the conversion of methanol to formaldehyde were determined in a continuous flow pilot plant (Fig. 1). The reactor was stainless steel (316-heat resistant), length $800 \mathrm{~mm}$, inside diameter, $19 \mathrm{~mm}$. Heaters were in the form of four separately heat-controlled block shells. The reactor was packed with $110 \mathrm{ml}$ of the catalyst between two layers of inert material such as glass, inert alumina or porcelene balls. Test conditions were: reactor temperature 200 to $35^{\circ} \mathrm{C}\left(473-623^{\circ} \mathrm{K}\right)$; pressure, $10 \mathrm{~atm}(1013 \mathrm{kPa})$; flow rate, $15.858 \mathrm{~cm}^{3} / \mathrm{s}$; methanol, $5.5 \%$ by volume in oxygen.

In a typical run the reactor was purged with $\mathrm{N}_{2}$ gas and the temperature raised. After establishing steady state conditions, the reaction was started; the methanol-oxygen mixture was pumped upwards into the reactor. The products were passed through three traps in series filled with water cooled to $278^{\circ} \mathrm{K}$ to absorb formaldehyde, unreacted methanol and side products.

Analysis of the reaction products was carried out periodically after two hours collection of the samples. Formaldehyde analysis was done by treating it with hypodidate solution which was prepared immediately by reacting iodine and $\mathrm{NaOH}$. Then $\mathrm{H}_{2} \mathrm{SO}_{4}$ was added to liberate iodine again some reacted with $\mathrm{HCHO}$ and the excess is backing titrated solution (Monti, et al. 1985). Unconverted methanol was determined gas chromatographically with a povpak type $\mathrm{T}$ column at $420^{\circ} \mathrm{K}$ (Monti, et al. 1985).

\section{Results and Discussion}

The results of this work has been tabulated in Table 1 . It can be seen that the Catalyst (3) (prepared by co-precipitation) is closest to the commercial catalyst in its composition and physical properties.

They are structurally similar as shown by their X-ray diffraction patterns (Fig. 2). The most obvious difference between the different preparations is the excess MoO3, which is greatest for catalyst (3) Table 1 . These preparations along with the commercial catalyst enable us to assess the effect of the molybdenum content on the properties of the catalysts. Excess molybdenum appears to have little effect on the catalyst density. However, the two catalysts with the highest molybdenum (catalyst (3) and the commercial catalyst) have the greatest surface areas, 
Table 1. Composition and properties of the catalysts

\section{Composition and Property}

\begin{tabular}{|c|c|c|c|c|}
\hline & Catalyst (1) & Catalyst (2) & Catalyst (3) & Commero \\
\hline & $\begin{array}{l}\text { Kneading and } \\
\text { evaporation }\end{array}$ & $\begin{array}{l}\text { Precipitation } \\
\text { and } \\
\text { concentration }\end{array}$ & $\begin{array}{l}\text { Co-precipitation } \\
\text { and filtration }\end{array}$ & Cutimerce \\
\hline \multicolumn{5}{|l|}{ Composition/wt \% } \\
\hline $\mathrm{Fe}$ & 17.2 & 15.6 & 13.8 & 14.0 \\
\hline Mo & 50.2 & 51.7 & 53.4 & 53.0 \\
\hline $\mathrm{Fe}_{2} \mathrm{O}_{3}$ & 24.6 & 22.3 & 19.7 & 20.0 \\
\hline $\mathrm{MoO}_{3}$ & 75.4 & 77.7 & 80.2 & 80.0 \\
\hline $\mathrm{MoO}_{3}$ excess $/ \mathrm{wt}-\%^{\mathrm{c}}$ & 2.32 & 4.57 & 7.12 & 6.52 \\
\hline Mo/Fe atomic ratio & 1.70 & 1.93 & 2.25 & 2.20 \\
\hline $\begin{array}{l}\mathrm{Fe} /(\mathrm{Fe}+\mathrm{Mo}) \text { mole } \\
\text { fraction }\end{array}$ & 0.371 & 0.341 & 0.308 & 0.312 \\
\hline Colour & Yellow green & Yellow green & Yellow & Yellow \\
\hline Pellet size/cm & $0.9 \times 0.9$ & $0.9 \times 0.9$ & $0.9 \times 0.9$ & $0.45 \times 0.4$ \\
\hline Surface area $/ \mathrm{m}^{2} \mathrm{~g}^{-1}$ & 283 & 282 & 287 & 290 \\
\hline Pore volume $/ \mathrm{cm}^{3} \mathrm{~g}^{-1}$ & 0.28 & 0.30 & 0.40 & 0.35 \\
\hline Solid density/g $\mathrm{cm}^{-3 \mathrm{~b}}$ & 0.53 & 0.50 & 0.52 & 0.50 \\
\hline Bulk density/g $\mathrm{cm}^{-3 \mathrm{~b}}$ & 1.05 & 1.05 & 1.05 & 1.10 \\
\hline Hardness $/ 10^{5}$ dyne & 1.70 & 1.63 & 2.1 & 2.3 \\
\hline
\end{tabular}

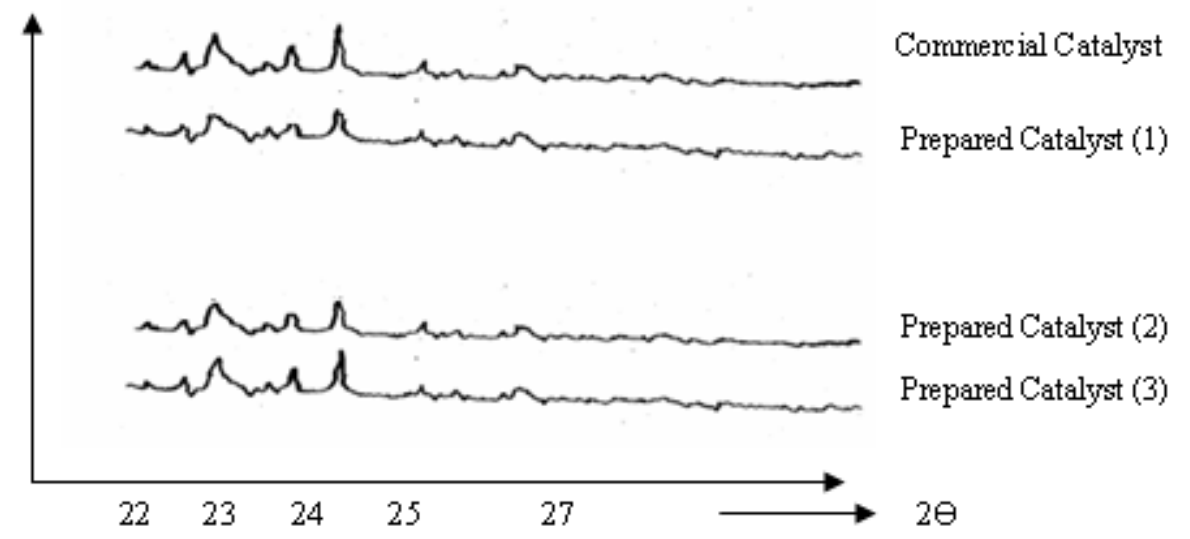

Figure 2. X-Ray powder diffraction patterns of the catalysts

pore volumes and hardness.

The activity and selectivity in the oxidation of methanol to formaldehyde are calculated. The activity is the percentage of methanol converted to all products and the selectivity as the fraction of formaldehyde in the products, ie. formaldehyde produced divided by methanol converted. The aim in the catalysis is to maximize both the conversion and the selectivity at the lowest reaction temperature. Activities and selectivities are shown plotted against the reaction temperature in Fig. 3. The behaviors of the catalyst (3) are similar to that of the commercial catalyst. The activities of all catalysts rise with rising temperature and converge to roughly the same conversion at $598^{\circ} \mathrm{K}$. The significant distinction between the catalysts is in the selectivity which passes through a maximum at $573^{\circ} \mathrm{K}$ with the commercial and the co-precipitated catalysts having the highest selectivities. The optimum combination of conversion and selectivity is provided by catalyst (3) and the commercial catalyst. Figure 4 shows that the variation of surface area (which for our catalysts is $\pm 1 \%$ ) is not significant.

The prepared catalytic results are consistent with the literature, eg. (Soares, et al. 2001); activities at constant surface area tending to the same value independently on the iron (or molybdenum) content of the catalyst; selectivities passing through a maximum with increasing reaction temperature. The implication of this observation is that during use, the catalyst surface stabilizes to a certain composition during the period of $12 \mathrm{~h}$ required to attain the higher tem- 

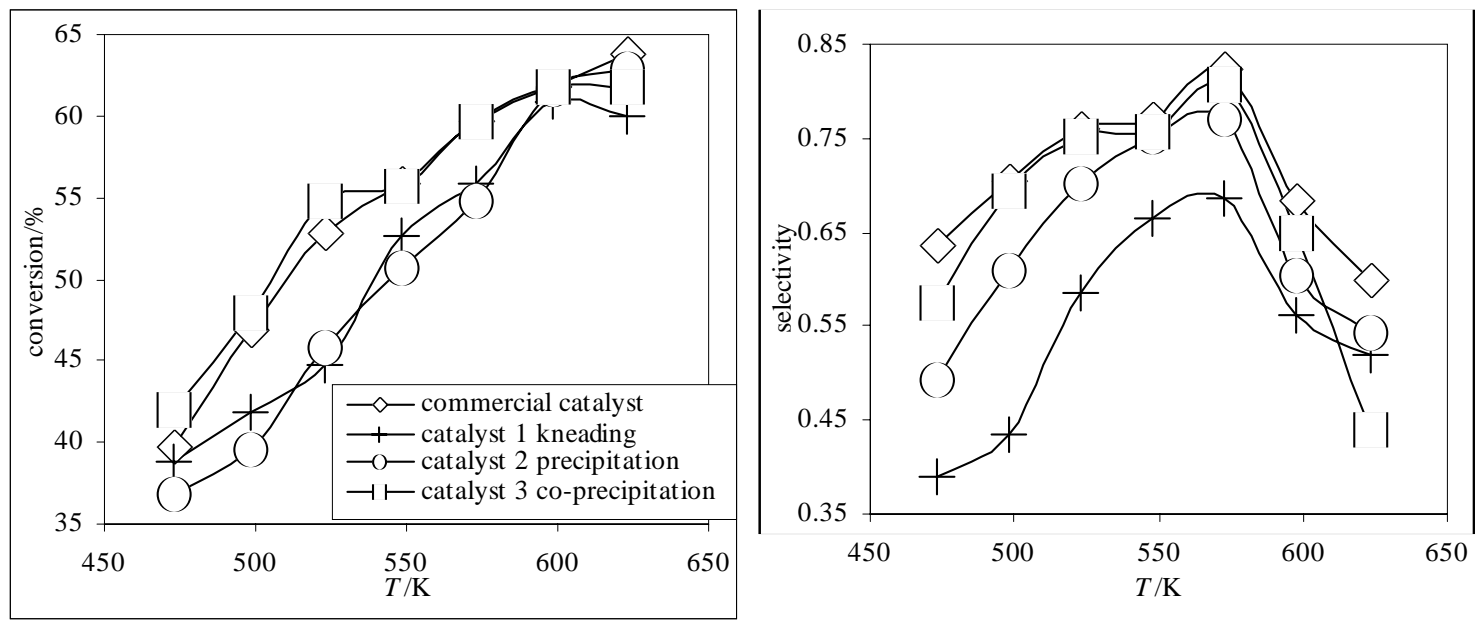

Figure 3. Conversion (left) and selectivity (right) in conversion of methanol to formaldehyde

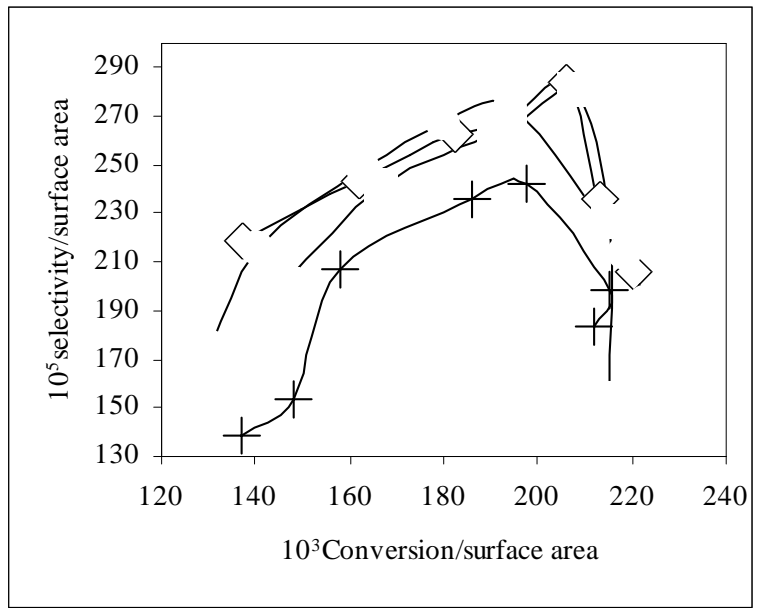

Figure 4. Selectivity towards formaldehyde vs. conversion of methanol for iron molybdate catalysts. Selectivities and activities are normalized to unit surface area. Symbols as in Figure 3

peratures as shown in Fig. 3.

The effect of catalyst composition on the activity and selectivity has been discussed with reference to the investigation results and literature data (Wachs, et al. 2000). The above discussion is in terms of excess of $\mathrm{MoO}_{3}$. However, since $\mathrm{MoO}_{3}$ is in excess it would seem logical to express the variation of catalyst composition in terms of iron added (or not) to molybdenum, ie. the Fe/Mo ratio or the $\mathrm{Fe} /(\mathrm{Fe}+\mathrm{Mo})$ mole fraction as for other two-component catalysts, for example, the cobalt-promoted molybdenum disulfide based hydrodesulphurization catalyst. It has been observed that in the model of two-component $\mathrm{MoO}_{3}$ based selective oxidation catalysts; the second component, e.g. bismuth or cobalt as in the selective oxidation of propene, enhances the activity by promoting the slow step, the first $\mathrm{C}-\mathrm{H}$ bond breaking and the abstraction of $\mathrm{H}$. The first step is catalyzed by the more basic oxide, $\mathrm{Bi}_{2} \mathrm{O}_{3}$ or $\mathrm{CoO}$. We imagine that the greater basicity (or nucleophilicity) of the oxide of the basic oxide favors interac- tion of the hydrogen of a methyl group with oxide of the catalyst, and subsequent proton transfer to oxide. When we interpret the data correctly we shall see that the iron molybdate catalyst fits into this pattern. It can be seen also that there was a synergy between iron and molybdenum.

Unfortunately most researchers have not studied a wide range of $\mathrm{Fe} / \mathrm{Mo}$ compositions. However, it can fit certain patent data (Wachs, et al. 2000) to these data and thereby examine a wider range of compositions. Activities and selectivities so obtained are plotted in Fig. 5. Activities and selectivities have been normalized of the commercial catalyst at $573^{\circ} \mathrm{K}$ to the values for a similar commercial catalyst reported (Wachs, et al. 2000). For the activities it can be seen a typical volcano curve, the activity rising to a maximum value as iron is added to $\mathrm{MoO}_{3}$ and then dropping off. This behavior demonstrates synergy between iron and molybdenum. Beyond an iron mole fraction of 0.4 the activity begins to increase as $\mathrm{Fe}_{2} \mathrm{O}_{3}$ takes over. The selectivity to formaldehyde is more or less constant until an iron mole fraction of 0.3 is reached. The selectivity then drops as $\mathrm{Fe}_{2} \mathrm{O}_{3}$ becomes dominant. This behavior indicates that the selective catalyst is $\mathrm{MoO}_{3}$. Iron is an activity promoter.

We can now understand more clearly how the iron molybdate catalyst works as also reported in the literature (Bowker, et al. 2002). It can be suggested here that the crucial feature is to have $\mathrm{Fe}^{3+}$ well dispersed in $\mathrm{MoO}_{3}$. The formation of patches or particles of $\mathrm{Fe}_{2} \mathrm{O}_{3}$ is to be avoided if the selectivity to formaldehyde is to be preserved. The loss of selectivity in Fig. 5 at higher iron levels is evidently associated with formatted of $\mathrm{Fe}_{2} \mathrm{O}_{3}$. The loss of activity may be attributed to the blocking of $\mathrm{MoO}_{3}$ sites. The dramatic loss of activity and selectivity seen in Fig. 5 represents a changeover from the preference for formaldehyde on a $\mathrm{MoO}_{3}$ catalyst to total oxidation on $\mathrm{Fe}_{2} \mathrm{O}_{3}$. The selective catalysis has often been associated with a definite compound, iron (III) molybdate, $\mathrm{Fe}_{2}\left(\mathrm{MoO}_{4}\right)_{3}$. The formation of iron (III) molybdate seems inevitable in the synthesis of the catalyst. A structural fea- 

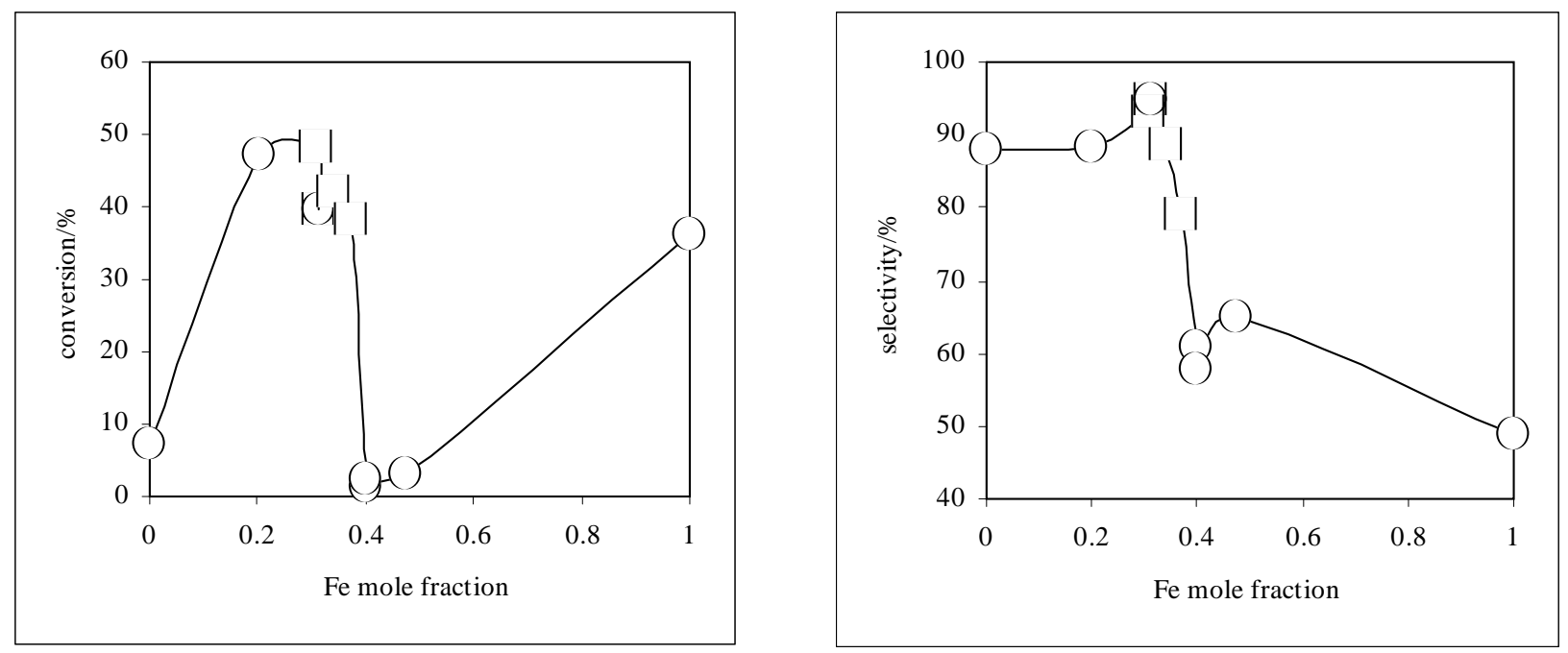

\section{Figure 5. Conversion and selectivity at $573 \mathrm{~K}$ for our iron molybdate catalysts (squares) and other reported} catalysts (23) (circles) vs. mole fraction of iron

ture of iron (III) molybdate is the presence of -O-Mo-OFe-O-Mo-O- groups. Such structures have been regarded as to represent the well dispersed $\mathrm{Fe}^{3+}$. The presence of iron(III) molybdate in our catalysts is indicated from the $\mathrm{X}$-ray diffraction pattern. It had been deducted from the catalytic data that the catalysts with the highest activity and selectivity (commercial catalyst and catalyst 3 ) have the most favorable $\mathrm{Fe}^{3+}$ dispersion. The method of preparation of catalyst (3) (co-precipitation and filtration) is likely lead to a better dispersion of $\mathrm{Fe}^{3+}$ and the least likely formation of $\mathrm{Fe}_{2} \mathrm{O}_{3}$ patches.

\section{Conclusions}

It was observed that the iron (III) molybdate catalyst prepared in the present work by co-precipitation and filtration method has a higher selectivity towards formaldehyde in methanol oxidation comparable with a commercial catalyst. Catalysts prepared by reacting iron (III) and molybdate by kneading or precipitation followed by evaporation, omitting a filtration stage, are less active and less selective. By combining our catalytic data with data from the patent literature, it can be demonstrated that the synergy between iron and molybdenum in regard to methanol oxidation to formaldehyde with an optimum composition corresponding to an iron mole fraction of 0.2-0.3. The selectivity to formaldehyde is practically constant to an iron mole fraction 0.3 and then decreases at higher iron concentrations. If we think of the iron component as the activity promoter then the iron molybdate catalysts can be related to other two-component $\mathrm{MoO}_{3}$-based selective oxidation catalysts, eg. bismuth and cobalt molybdate. The iron oxide then functions as a relatively basic oxide abstracting, in the rate-controlling step, a proton from the methyl of a bound methoxy group of chemisorbed methanol. The proposed crucial feature of the iron(III) molybdate catalyst is the presence of -O-Mo-O-Fe-O-Mo-
O- groups as found in the compound $\mathrm{Fe}_{2}\left(\mathrm{MoO}_{4}\right)_{3}$ and for $\mathrm{Fe}^{3+}$ which is generally the well dispersed in $\mathrm{MoO}_{3}$. At the higher iron (III) concentrations the loss of selectivity is due to the presence of iron oxide patches or particles which catalyze the total oxidation of methanol and the loss of activity to blocking of molybdenum sites.

\section{References}

Boreskov, G.K., Delmon, B., Jacobs, P.A. and Poncelet, G., 1976, "In Preparation of Catalysts," Elsevier, Amsterdam, pp. 223-227.

Bowker, M., Holroyd, R., Elliott, A., Morrall, P., Alouche, A., Entwistle, C. and Toerncrona, A., 2002, "Catalysis Letters," Vol. 83, pp. 165-176.

Catal, T., 1997, "Mars van Krevelen,” Vol. 34, p. 40.

Dia Kov. V., Larfarge, D. and Varma, A., 2001, "Methanol Oxidation Dehydrogenation in a Catalytic Packed Bed Membrane Reactor", Catalysis Today, Vol. 76, pp. 159-167.

Diakov, V., Blackwell, B. and Varmay, A., 2002, "Methanol Oxidative Dehydrogenation in a Catalytic Peaked-Ped Membrane Reactor; Experimental and Model," Chemical Engineering Science, Vol. 57, pp. 1563-1569.

Farrauto, R.J. and Bartholomew, C.H., 1997, "Fundamentals of Industrial Catalytic Processes,“ Blackie Academic and Professional, London, p. 489.

Ivanov, K., Mitov, I. and Kruster., "Selective Oxidation of Methanol on Fe-Mo-W Catalysts," J. of Alloys and Compounds, Vol. 309(14), pp. 57-60.

Jia-Liang, Li., Wei-Lin Dai., Ky Dong, Jing- Fa Domg, 2000, " A New Silver-Containing Ceramics for Catalytic Oxidation of Methanol to Formldehyde," Materials Letters, Vol. 44(3-4), pp. 233-236.

Le Pag, J.F. and Miquel, J.In., Delmon, B., Jacobs, P.A. and Poncelt, G., 1976, "Preparation of Catalysts," 
Elsevier Amsterdam, pp. 39-43.

Le Page, J.F., 1987, "Applied Heterogeneous Catalysis," Technip Paris.

McCarron, E.M. III., Sleight, A.W., Mitchell, P.C.H. and Sykes. A.G., 1986, "The Chemistry and Uses of Molybdenum," Proceedings of the Climax Fifth International Conference, Polyhedron Synposia-inPrint Number 2, Pergamon Press, Oxford, p. 129.

Monti, D., Reller, A., and Baiker, A., 1985, "Methanol Oxidation on $\mathrm{K}_{2} \mathrm{SO}_{4}$ - Promoted Vanadium Pentoxide; Activity, Reducibility and Structure of Catalysts,” J. of Catalysis, Vol. 93, pp. 360-367.

Powder Diffraction File, 1978, “Alphabetical Listing," Swarthmore Pennsylvania.

Satter Filled and Charles N., 1980, "Heterogenous Catalysis in Practice," Mc Graw-Hill, Inc., New York.

Soares A.P.V, Portela M.F.,Kiennemann A.,Hilaire L. and Millet J.M.M., 2001, "Iron Molybdate Catalysts for Methanol to Formaldehyde Oxidation; Effect of Mo excess on Catalystic Behaviour," Applied Catalysis, Vol. 206, pp. 221-229.

Soares A.P.V., Portela M.F. and Kiennemanu A., 2001,
"Iron Molybdate Catalysts for Methanol to form Aldehyde Oxidation; Effect of Mo Excess on the Deactivation behaviour," Catalysis Communications, Vol. 2, pp. 159-164.

Soares, A.P.V., Portela, M.F., Kiennemann, A. and Hilaire, L., 2003, "Chemical Engineering Science," Vol. 58, pp. 1315-1322.

Soares, A.P.V., Portela, M.F. and Kiennemann, A., 1997, "3rd World Congress on Oxidation Catalysis," Vol. 110, 807-816.

The Formaldehyde Council, Inc. (FCI): http://www.formaldehyde.org.

Topsoe, H., Clausen, B.S., Burriescin, Candia R. and Morups, 1976, "In preparation of Catalysts," Elsevier, Amsterdam, pp. 479-487.

Wachs I.E.,2003, "Extending Surface Science Studies to Industrial Reaction Conditions; Mechanism and Kinetics of Methanol Oxidation over Silver Surface,” Surface Science, Vol. 544, pp. 1-4.

Wachs, I.E. and Briand, L.E., 2000, “US Patent 6037290," Lehigh University, Bethlehem, PA, USA.

Wang. C.T. and Willey, R.J., 2001, “Journal of Catalysis,” Vol. 202, pp. 211-219. 